\title{
Monitoring drug safety in Astrakhan, Russia
}

\author{
O.O. Kirilochev*, I.P. Dorfman and A.R. Umerova \\ Astrakhan State Medical University, Clinical Pharmacology Department, Astrakhan, Russia \\ * Corresponding author. E-mail: kirilochev@gmail.com
}

BACKGROUND: The problem of drug safety will never disappear as new drugs are delivered in increasing numbers. They have high biological activity and adverse drug reactions (ADR) [1]. Currently, adverse drug reactions are the fourth leading cause of death for patients.

There are databases of ADRs (Vigibase, Eudravigilance), but we know that ADR manifestations may vary in different countries and regions, due to the demographic, genetic characteristics of the population and the quality of manufactured drugs [2]. In this regard, the study of the ADR at the regional level is very relevant. We aimed to optimize the work on monitoring drug safety in Astrakhan region through pharmacoepidemiological research and development of computer database for analysis of information coming to the center for drug safety monitoring (CDSM).

\section{OBJECTIVES:}

1. To study the rates of ADR reporting and the structure in the Astrakhan region at the regional center for drug safety monitoring.

2. To analyze the outcomes of registered adverse drug reactions.

3. To determine the causality of adverse drug reactions.

4. To identify reports on the ineffectiveness of drugs.

5. To analyze the rates and structure of ADR reporting for drugs prescribed off-label.

METHODS: We studied spontaneous adverse event reporting. The adverse event reports received by the regional CDSM for the period of 2010 to 2014 was analyzed. The groups of drugs were categorized according by Anatomical Therapeutic Chemical classification system. The data were analyzed using Microsoft Office Excel. The likelihood of whether an ADR was actually due to the drugs was assessed with the Naranjo algorithm.

RESULTS: The analysis of the results showed that the establishment of the CDSM in September 2010, contributed to improvement of drug safety monitoring in health facilities of the region. Noteworthy was the increasing the number of adverse event reports in 2011 and 2012, compared with the beginning of the year 2010, when the CDSM was not yet functioning.

The decrease of adverse event reporting in 2013 and 2014 was due to the fact that doctors in the region had access to better ADR drug information. Along with the increasing number of adverse event reporting we also noted the increase in the number of health facilities that monitored drug safety. The number of health facilities that reported, doubled from 2010 to 2014. We observed the increase in the number of adverse event reports submitted by pharmaceutical companies. General anti-infective drugs for systemic use (class J) were the most common cause of all registered ADRs (44\%). Drugs 
for treatment of tuberculosis (group J04A) were the cause of adverse drug reactions in $34 \%$ of reports. ADRs associated with drugs used for treating diseases of cardiovascular system accounted for $16 \%$ of case-reports; drugs belonging to the group of Alimentary tract and metabolism (class A) and to the group of Nervous System (class A) were reported to cause ADRs in 10\% of cases each. Type A adverse drug reactions, which are usually a consequence of a drug's primary pharmacological effect, were detected in $45 \%$ of cases. These reactions were often registered for drugs affecting cardiovascular system (class C), nervous system (class N), dlood and blood forming organs (class B). Type B ADRs were reported in $54 \%$ of cases. These were "idiosyncratic" reactions, which could not be predicted on the basis of the drug's main pharmacological action, were not dose-related and were severe [3]. The most frequent cause of type B adverse drug reactions was the General anti-infective medicines for systemic use (Class J). The fatality rate associated with ADRs was $0.3 \%$. Type A adverse drug reactions resulted in death in $38 \%$ of cases. Type B ADR (anaphylactic shock) accounted for $62 \%$ of the patient's deaths. The Naranjo scale determined the causality of ADRs. The "definite" ADRs were detected in 14\% of reports, "probable" in $47 \%$, and "possible" - in 39\% of cases. The rate of reporting associated with ineffectiveness of drugs amounted to $1 \%$. Most often the lack of therapeutic effect was reported in patients receiving drugs of class C (Cardiovascular system, 31\% of all cases of inefficiency of drugs). These drugs were used in accordance with their official instructions for use. The proportion of ADR reports for drugs prescribed off-label was $1.4 \%$.

CONCLUSIONS: The results substantiate the need to continue drug safety monitoring in the Astrakhan region. We plan to further improve the software for ADR analysis.

Keywords: Drug safety, monitoring, adverse drug reaction, regional reporting system, centre for drug safety monitoring

\section{Conflict of interest statement: None.}

\section{References}

[1] Zborovsky AB, Turenkov IN, Belousov UB. Side effects of drugs. Moscow: Medicine Information Agency Ltd; 2008.

[2] Kostyleva MN, Belousov UB, Gracianskaya AN, Postnikov SS. Safety assessment of drug therapy in clinical practice. Pharmacoeconomics. 2014;1:26-30.

[3] Ritter J, Lewis L, Mant T, Ferro A. A Textbook of Clinical Pharmacology and Therapeutics. London: Hodder Arnold; 2008. 